Copyright by the American Physical Society. Lee, T. K.; Zhang, F. C., "Extended and localized states in the periodic Anderson model," Phys. Rev. B 34, 8114 DOI: http://dx.doi.org/10.1103/PhysRevB.34.8114

\title{
Extended and localized states in the periodic Anderson model
}

\author{
T. K. Lee \\ Department of Physics, Virginia Polytechnic Institute and State University, Blacksburg, Virginia 24061 \\ F. C. Zhang* \\ Center for Theoretical Physics, Department of Physics and Astronomy, University of Maryland, \\ College Park, Maryland 20742
}

(Received 2 June 1986)

\begin{abstract}
The renormalized quasiparticle states are derived for a periodic Anderson model with a general hybridization matrix element between conduction electrons with two degrees of freedom and $f$ electrons with $N$ degrees of freedom. Only two out of $N$ local $f$ states form the extended quasiparticle bands while $N-2$ localized states remain. As an illustration we show that the self-energy due to the Kondo effect produces quasiparticle bands and a gap as obtained in the Kondo-boson approach.
\end{abstract}

Since the earlier work by Martin ${ }^{1}$ the puzzle regarding the band structure of the periodic Anderson model has become increasingly interesting. Recently several groups have used either the Kondo-boson approach ${ }^{2-4}$ or the diagrammatic method ${ }^{5}$ to derive the coherent quasiparticle bands. But for simplicity they study the so-called $S U(N)$ model where both conduction electrons and $f$ electrons have the same number of degenerate states. And within the mean-field theory of the Kondo-boson approach the quasiparticle gap would be formed at Kondo temperature.

In this paper, we report the derivation of quasiparticle bands and localized states for the periodic Anderson model with a general hybridization matrix element $V_{\mathbf{k} \sigma m}$ between conduction electrons $\mathbf{k} \sigma$ and localized $f$ electrons $\mathrm{Jm}$. There are total $N=2 J+1$ degrees of freedom for $f$ electrons and only two for conduction electrons.

We find out that only two out of the $N$ channels of the $f$ electron hybridize with conduction electrons and become extended states. There are $N-2$ localized states. These results are in agreement with the variational approach by Rice and Ueda, ${ }^{6}$ who studied the model with the simpler hybridization matrix element.

We consider the Anderson-lattice model in the Kondo regime, namely, where the Kondo effect is more important than the Ruderman-Kittel-Kasuya-Yosida interaction. In this regime, we may start with the results for the singleimpurity Anderson model and treat the intersite coupling perturbatively. By using Dyson's equation we derive the intersite $f$-electron and conduction-electron Green's functions in terms of the single-ion on-site $f$ Green's function. The vertex correction is neglected in the derivation. The vertex will be discussed elsewhere. ${ }^{7}$ To illustrate the result we use the hybridization matrix element obtained by Coqblin and Schrieffer ${ }^{8}$ for the Ce ion with $J=\frac{5}{2}$.

The single-impurity $f$-electron Green's function can be evaluated by using the diagrammatic method ${ }^{9-11}$ or the functional-integral technique. ${ }^{12,13}$ We then obtain the re- normalized conduction-electron and $f$-electron states, showing a gap at zero temperature, which is ensured by the Friedel sum rule proved by Langreth. ${ }^{14}$ In contrast to the mean-field theory of the Kondo-boson approach where the gap is formed abruptly at the Kondo temperature, the gap in our theory will be formed only at zero temperature and it is a slowly evolving process.

We study a periodic Anderson model with a Hamiltonian

$$
\begin{aligned}
H_{A}= & \sum_{\mathbf{k}, \sigma} \varepsilon_{\mathbf{k}} C_{\mathbf{k} \sigma}^{\dagger} C_{\mathbf{k} \sigma}+\sum_{m, j} \varepsilon_{f} X_{m j}^{\dagger} X_{m j} \\
& +\frac{1}{\sqrt{N_{s}}} \sum_{\mathbf{k}, \sigma, m, j}\left(V_{\mathbf{k} \sigma m} e^{i \mathbf{k} \cdot \mathbf{R}_{j}} C_{\mathbf{k} \sigma}^{\dagger} X_{m j}+\text { H.c. }\right) .
\end{aligned}
$$

The conduction-band dispersion is chosen to be linear in the range from $-D$ to $+D$. The projection operator on site $j, X_{m j}$, changes the localized $f$ configuration from $\left|f^{1}, J m\right\rangle$ to $\left|f^{0}\right\rangle$. In the Kondo limit, the bare $f$ level has energy $\varepsilon_{f}<0$, and the on-site Coulomb repulsion $U$ has been set to infinity.

The only symmetry property of the hybridization matrix element $V_{\text {kom }}$ that will be used below is

$$
\int \frac{d \Omega_{k}}{4 \pi} \sum_{\sigma} V_{\mathbf{k} \sigma m}^{*} V_{\mathbf{k} \sigma m^{\prime}}=V_{k}^{2} \delta_{m m^{\prime}}
$$

where $V_{k}$ is independent of the angle of $\mathbf{k}$. Equation (2) is used extensively in the single-impurity model. ${ }^{9,15}$

Let us first define the intersite $f$ Green's function,

$$
g_{m m^{\prime}}\left(R_{i j}, \tau\right)=-\left\langle T_{\tau} X_{m j}(\tau) X_{m^{\prime} j^{\prime}}^{\dagger}\right\rangle
$$

A simple way to derive the quasiparticle bands is to connect the $f$-electron Green's function on every site with a conduction-electron Green's function.

Dyson's equation for the Fourier transform of $g_{m m}$ can be easily written down; it is of the form

$$
g_{m m^{\prime}}\left(R_{i j}, \omega\right)=g_{m}^{0} \delta_{i j} \delta_{m m^{\prime}}+\sum_{l, m_{1}} g_{m}^{0}\left(\frac{1}{N_{s}} \sum_{\mathbf{k}, \sigma} V_{\mathbf{k} \sigma m}^{*} V_{\mathbf{k} \sigma m_{1}} g_{c}(\mathbf{k} \sigma) e^{i \mathbf{k} \cdot \mathbf{R}_{l l}}\right)\left(1-\delta_{i l}\right) g_{m_{1} m^{\prime}}\left(R_{l j}, \omega\right)
$$


where $g_{m}^{0}$ is the single-impurity $f$ Green's function in frequency space, and the unrenormalized conduction-electron Green's function is

$$
g_{c}(\mathbf{k} \sigma)=\left(i \omega-\varepsilon_{\mathbf{k}}\right)^{-1}
$$

We emphasize that the vertex correction is not inlcuded in Eq. (4).

Taking the Fourier transform of Eq. (4), we obtain

$g_{m m^{\prime}}(\mathbf{k})=\tilde{g}_{m}\left(\delta_{m m^{\prime}}+\sum_{\sigma} V_{\mathbf{k} \sigma m}^{*} g_{c}(\mathbf{k} \sigma) \sum_{m_{1}} V_{\mathbf{k} \sigma m_{1}} g_{m_{1} m^{\prime}}(\mathbf{k})\right)$

where

$$
\tilde{g}_{m}=\left[\left(g_{m}^{0}\right)^{-1}+\Gamma(\omega)\right]^{-1},
$$

and

$$
\Gamma(\omega)=\frac{1}{N_{s}} \sum_{\mathbf{k}, \sigma}\left|V_{\mathbf{k} \sigma m}\right|^{2} g_{c}(\mathbf{k} \sigma) .
$$

We have used Eq. (2) in the derivation given above. $\Gamma(\omega)$ is independent of quantum number $m$, and it can be easily evaluated:

$$
\Gamma(\omega)=-i \pi \Delta \operatorname{sgn} \omega
$$

where

$$
\Delta=\frac{1}{N_{s}} \sum_{\mathbf{k}, \sigma}\left|V_{\mathbf{k} \sigma m}\right|^{2} \delta\left(\omega-\varepsilon_{\mathbf{k} \sigma}\right) .
$$

Equation (6) is a matrix equation and can be easily solved. The result is

$$
g_{m m^{\prime}}(\mathbf{k})=\tilde{g}_{m} \delta_{m m^{\prime}}+\tilde{g}_{m} \tilde{g}_{m^{\prime}} \sum_{\sigma} V_{\mathbf{k} \sigma m}^{*} g_{c}(\mathbf{k} \sigma)\left[V_{\mathbf{k} \sigma m^{\prime}}(1-\gamma-\sigma,-\sigma)+V_{\mathbf{k},-\sigma, m^{\prime}} \gamma_{\sigma,-\sigma}\right][\operatorname{det}(\underline{1}-\underline{\gamma})]^{-1},
$$

where the matrix element of the $2 \times 2$ matrix $\underline{\gamma}$ is given by

$$
\gamma_{\sigma \sigma^{\prime}}=\sum_{m} V_{\mathbf{k} \sigma m} \tilde{g}_{m} V_{\mathbf{k} \sigma^{\prime} m}^{*} g_{c}\left(\mathbf{k} \sigma^{\prime}\right) \text {. }
$$

The renormalized conduction-electron Green's function is simply related to $g_{m m^{\prime}}(\mathbf{k})$, i.e.,

$$
\begin{aligned}
\tilde{g}_{c}\left(\mathbf{k} \sigma \sigma^{\prime}\right)= & g_{c}(\mathbf{k} \sigma) \delta_{\sigma \sigma^{\prime}} \\
& +g_{c}(\mathbf{k} \sigma) \sum_{m, m^{\prime}} V_{\mathbf{k} \sigma m} g_{m m^{\prime}}(\mathbf{k}) V_{\mathbf{k} \sigma^{\prime} m^{\prime}}^{*} g_{c}\left(\mathbf{k} \sigma^{\prime}\right)
\end{aligned}
$$

Substitution of Eq. (11) into (13) yields

$$
\tilde{g}_{c}\left(\mathbf{k}, \sigma \sigma^{\prime}\right)=g_{c}(\mathbf{k} \sigma)(\underline{1}-\underline{\gamma})_{\sigma \sigma^{\prime}}^{-1} \text {. }
$$

We emphasize that, starting with the single-ion $f$ Green's function, only the symmetry property of Eq. (2) is used in the derivation.

Equation (11) implies that $m$ is no longer a good quantum number for $f$ electrons. A very important result associated with $g_{m m} \cdot(\mathbf{k})$ is obtained by taking the sum,

$$
\sum_{m} g_{m m}(\mathbf{k})=(N-2) \tilde{g}_{m}+\tilde{g}_{m} \operatorname{Tr}\left\{[1-\underline{\gamma}(\mathbf{k})]^{-1}\right\}
$$

Equation (15) can be used to calculate the total renormalized $f$-electron density of states. Since the function $\tilde{g}_{m}$ is independent of $\mathbf{k}$, Eqs. (7) and (15) show that there are $N-2$ localized levels and two extended bands for $f$ electrons. Even if there exists a gap for the extended bands, as an example illustrated below, the localized states will fill the gap at finite temperatures. Thus the density of states is similar to a highly doped semiconductor. We note that the procedure used here will lead to the exact result for the $U=0$ periodic Anderson model.

Equations (12) and (15) can be further simplified for the systems with the following symmetry property

$$
\sum_{m} V_{\mathbf{k} \sigma m}^{*} V_{\mathbf{k} \sigma^{\prime} m}=\delta_{\sigma \sigma^{\prime}} \frac{N}{2} V_{k}^{2} \text {. }
$$

This equation is consistent with the hybridization matrix element obtained by Coqblin and Schrieffer ${ }^{8}$ for $\mathrm{Ce}$, where

$$
\begin{aligned}
V_{\mathbf{k} \sigma m}=V_{k} \sqrt{4 \pi}(-i)^{3}\{ & \delta_{\sigma, 1 / 2} \beta_{m}\left[Y_{3}^{m-1 / 2}\left(\Omega_{\mathbf{k}}\right)\right]^{*} \\
& \left.+\delta_{\sigma,-1 / 2} \alpha_{m}\left[Y_{3}^{m+1 / 2}\left(\Omega_{\mathbf{k}}\right)\right]^{*}\right\} .
\end{aligned}
$$

$Y_{3}^{m \pm 1 / 2}\left(\Omega_{k}\right)$ is the $l=3$ spherical harmonic function for the wave vector $\mathbf{k}$. The quantity $V_{k}$ only depends on the magnitude of $\mathbf{k}$. In Eq. (17), $\alpha_{m}$ and $\beta_{m}$ are the ClebschGordon coefficients; they are given by

$$
\alpha_{m}=\left(\frac{7+2 m}{14}\right)^{1 / 2} \text { and } \beta_{m}=\left(\frac{7-2 m}{14}\right)^{1 / 2} \text {. }
$$

In the absence of magnetic field and by using Eq. (16), $\gamma_{\sigma \sigma^{\prime}}$ defined by Eq. (12) is simplified to the form

$$
\gamma_{\sigma \sigma^{\prime}}(\mathbf{k})=\delta_{\sigma \sigma^{\prime}} \frac{N}{2} V_{k}^{2} \tilde{g}_{m} g_{c}(\mathbf{k} \sigma)
$$

The renormalized conduction-electron Green's function now becomes the form

$$
\tilde{g}_{c}\left(\mathbf{k} \sigma \sigma^{\prime}\right)=\delta_{\sigma \sigma^{\prime}}\left(g_{c}(\mathbf{k} \sigma)^{-1}-\frac{N}{2} V_{k}^{2} \tilde{g}_{m}\right)^{-1} .
$$

The $f$-electron Green's function (11) is simplified to the form

$g_{m m^{\prime}}(\mathbf{k})=\tilde{g}_{m}\left(\delta_{m m^{\prime}}+\sum_{\sigma} \frac{V_{\mathbf{k} \sigma m}^{*} V_{\mathbf{k} \sigma m^{\prime}} g_{c}(\mathbf{k} \sigma)}{\tilde{g}_{m^{\prime}}^{-1}-(N / 2) V_{k}^{2} g_{c}(\mathbf{k} \sigma)}\right)$,

and Eq. (15) simply becomes

$$
\sum_{m} g_{m m}(\mathbf{k})=(N-2) \tilde{g}_{m}+\sum_{\sigma} \frac{1}{\tilde{g}_{m}^{-1}-(N / 2) V_{k}^{2} g_{c}(\mathbf{k} \sigma)} .
$$

To move forward we need the explicit form for the single impurity $f$-Green's function $g_{m}^{0}$. Using the $1 / N$ expansion and noncrossing approximation, we have shown in Ref. 10 that the $f$-electron Green's function is given by

$$
\begin{aligned}
g_{m}^{0}(i \omega)= & \frac{T_{0} / N \Delta}{i \omega-T_{0}+i(\operatorname{sgn} \omega) \pi T_{0} / N} \\
& +\frac{n_{f} / N}{i \omega-\varepsilon_{f}+i(\operatorname{sgn} \omega) \pi \Delta N},
\end{aligned}
$$


where $n_{f}$ is the $f$-electron occupation number. The first term gives the low-frequency Kondo resonance. The high-frequency charge excitation is given by the second term. Since we are interested in the electronic states near the Fermi surface, the second term can be safely neglected for this purpose. Then Eqs. (7) and (9) lead to

$$
\tilde{g}_{m}=T_{0} / N \Delta\left(i \omega-T_{0}\right) \text {. }
$$

The denominators of Eqs. (20) and (21) will be simply proportional to

$$
\begin{aligned}
& {\left[\left(g_{f}^{9}\right)^{-1}+\Gamma\right] g_{c}^{-1}(\mathbf{k} \sigma)-\frac{N}{2} V_{k}^{2}} \\
& \quad=\frac{N \Delta}{T_{0}}\left(\left(i \omega-T_{0}\right)\left(i \omega-\varepsilon_{\mathbf{k}}\right)-\frac{T_{0} V_{k}^{2}}{2 \Delta}\right) .
\end{aligned}
$$

Thus we have obtained two quasiparticle bands,

$$
E_{\mathbf{k}}^{ \pm}=\frac{1}{2}\left(\varepsilon_{\mathbf{k}}+T_{0}\right) \pm\left[\left(\frac{\varepsilon_{\mathbf{k}}-T_{0}}{2}\right)^{2}+\frac{T_{0} V_{k}^{2}}{2 \Delta}\right]^{1 / 2}
$$

These results agree with those of Rice and Ueda. ${ }^{6}$

Using the approximation discussed above, the Green's functions $\tilde{g}_{c}(\mathbf{k} \sigma)$ and $g_{m m}{ }^{\prime}(\mathbf{k})$ of Eqs. (20)-(22) become the form

$$
\begin{aligned}
& \tilde{g}_{c}(\mathbf{k} \sigma)=\frac{i \omega-T_{0}}{\left(i \omega-E_{\mathbf{k}}^{+}\right)\left(i \omega-E_{\mathbf{k}}^{-}\right)}, \\
& g_{m m^{\prime}}(\mathbf{k})=\frac{T_{0} / N \Delta}{i \omega-T_{0}}\left(\delta_{m m^{\prime}}+\frac{\sum_{\sigma} V_{\mathbf{k} \sigma m}^{*} V_{\mathbf{k} \sigma m^{\prime}} T_{0} / N \Delta}{\left(i \omega-E_{\mathbf{k}}^{+}\right)\left(i \omega-E_{\mathbf{k}}^{-}\right)}\right),
\end{aligned}
$$

and

$$
\begin{aligned}
\sum_{m} g_{m m}(\mathbf{k})= & (N-2) \frac{T_{0} / N \Delta}{i \omega-T_{0}} \\
& +\sum_{\sigma} \frac{\left(i \omega-\varepsilon_{\mathbf{k}}\right) T_{0} / N \Delta}{\left(i \omega-E_{\mathbf{k}}^{+}\right)\left(i \omega-E_{\mathbf{k}}^{-}\right)} .
\end{aligned}
$$

We note that the results of Eqs. (27)- (29) also can be obtained by making a mean-field approximation to $H_{A}$ in the Kondo-boson approach. But in mean-field theory, the symmetry is broken and the gap is formed at the Kondo temperature. On the other hand, in our derivation of Eqs. (27)-(29), we have neglected the correction to the width of the Kondo resonance; it has the form $\omega^{2} / T_{0}$ or $T^{2} / T_{0}$. Yet $\Gamma(\omega)$ of Eq. (9) has almost no correction. Thus the complete cancellation of the width in Eq. (24) most likely will not happen at temperatures of order $T_{0}$. Without invoking other higher-order processes and the charge fluc- tuation, our approach will predict that the formation of the gap or the buildup of the coherence is a continuous process and not a sudden phase transition as predicted by the mean-field theory.

As a consequence of the incomplete cancellation of the Kondo width, the $(N-2)$ localized states as shown in Eq. (22), will fill the gap. But at $T=0 \mathrm{~K}$ the periodicity prevails and it ensures that $\tilde{g}_{m}$ has no imaginary part at the Fermi energy. ${ }^{14}$

Objections might be raised for using the $f$ Green's function $g_{m}^{0}$ from an impurity model where the unrenormalized conduction-electron Green's function is used in calculating the self-energy. But in fact this result is not changed by using the renormalized conduction electronic bands of Eq. (27). A direct calculation with $\tilde{g}_{c}$ of Eq. (27) can easily verify this. Instead we shall give a simple physical argument. The characteristic Kondo temperature $T_{0}$ is determined from the ground-state energy $E_{0}=\varepsilon_{f}-T_{0}=N \Delta$ $\times \ln \left(T_{0} / D\right)$ in the leading $1 / N$ expansion, where the whole band contributes in the $\ln D$ term. The difference between the renormalized and unrenormalized bands are within a small energy range $T_{0}$ around Fermi level. The electronic wave function is also renormalized by a very small factor $\left(T_{0} / N \Delta\right)^{1 / 2}$. Thus a very small correction is expected.

In summary, using a general form of hybridization matrix element, we have derived the quasiparticle bands for the periodic Anderson model in the Kondo regime. We have shown that only two out of $N$ degrees of freedom of $f$ electrons form extended states. There are $N-2$ localized states. In our theory the on site $f$ Green's function determines all other Green's functions. The simplest approximation, by including only the Kondo effect, leads to familiar coherence bands ${ }^{2-6}$ and localized states. We have found no evidence for a phase transition from an incoherent to coherent state, as obtained by the mean-field theory of the Kondo-boson approach. Our theory predicts a continuous and gradual process of building up the coherence. The shortcoming associated with the perturbative approach presented in this paper seems to be the difficulty of consistently including the vertex correction in the Dyson's equation. This may be relatively easy in the Kondo-boson approach by including the Gaussian fluctuation.

At the last stage of the preparation of this paper, we received a copy of work by Z. Zou and P. W. Anderson ${ }^{16}$ prior to its publication. Essentially the same results for the hybridization of $f$ electrons are derived there using a different method.

This work is partly supported by the National Science Foundation, under Grant No. DMR-82-13768.
*Present address: Theoretische Physik, Eidgenössische Technische Hochschule, CH-8093 Zürich, Switzerland.

${ }^{1}$ R. M. Martin, Phys. Rev. Lett. 48, 362 (1982).

${ }^{2}$ N. Read, D. M. Newns, and S. Doniach, Phys. Rev. B 30, 384 (1984).

${ }^{3} \mathrm{P}$. Coleman, in Proceedings of the Eighth Taniguchi Symposium on the Theory of the Valence Fluctuation States, edited by T. Kasuya (Springer-Verlag, New York, in press).

${ }^{4} Z$. Tesanovic and O. T. Valls, Phys. Rev. B 34, 1918 (1986).

${ }^{5}$ N. Grewe and T. Pruschke (unpublished).

${ }^{6}$ T. M. Rice and K. Ueda, Phys. Rev. Lett. 55, 995 (1985).

${ }^{7}$ F. C. Zhang, T. K. Lee, and Z. B. Su (unpublished).

${ }^{8}$ B. Coqblin and J. R. Schrieffer, Phys. Rev. 185, 847 (1969).

${ }^{9}$ N. Grewe and H. Keiter, Phys. Rev. B 24, 4420 (1981). 
${ }^{10}$ T. K. Lee and F. C. Zhang, J. Appl. Phys. 55, 1936 (1984); F. C. Zhang and T. K. Lee, Phys. Rev. B 30, 1556 (1984).

${ }^{11}$ Y. Kuramoto, Z. Phys. B 53, 37 (1983).

${ }^{12}$ N. Read and D. M. Newns, J. Phys. C 16, 3273 (1983); P. Coleman, Phys. Rev. B 29, 3035 (1984).

${ }^{13}$ N. E. Bickers, D. C. Cox, and J. M. Wilkins, Phys. Rev. Lett. 54, 230 (1984); D. C. Cox (unpublished).

${ }^{14}$ D. C. Langreth, Phys. Rev. 150, 516 (1966). The exact can- cellation of the imaginary part of $\tilde{g}_{m}$ follows from the derivation by Langreth that the imaginary part of the self-energy of $g_{m}^{0}$ at Fermi surface is exactly equal to $\pi \Delta$. Because we have used the approximate form of $g_{m}^{0}$ given by Eq. (22), $\tilde{g}_{m}$ of Eq. (24) has no imaginary part at a range of low frequencies, not only at zero frequency.

${ }^{15}$ F. C. Zhang and T. K. Lee, Phys. Rev. B 28, 33 (1983).

${ }^{16} \mathrm{Z}$. Zou and P. W. Anderson (unpublished). 